\title{
A 3D finite element model of maximal grip loading in the human wrist
}

\author{
Magnús K. Gíslason ${ }^{1 *}$, David H. Nash ${ }^{1}$, Alexander Nicol ${ }^{1}$, Asimakis Kanellopoulos ${ }^{1}$, \\ Marc Bransby-Zachary ${ }^{2}$, Tim Hems ${ }^{3}$, Barrie Condon ${ }^{2}$, Benedict Stansfield ${ }^{4}$,
}

${ }^{1}$ Bioengineering Unit, Wolfson Building, University of Strathclyde, 106 Rottenrow, Glasgow,

G4 0NW, UK

${ }^{2}$ Southern General Hospital, Glasgow, UK

${ }^{3}$ Victoria Royal Infirmary, Glasgow, UK

${ }^{4}$ Glasgow Caledonian University, Glasgow, UK

${ }^{*}$ Corresponding author. Bioengineering Unit, Wolfson Building, University of Strathclyde, 106 Rottenrow, Glasgow, G4 0NW, UK. Tel.: +44 (0)141 548 3228; fax: $+44(0) 1415526098$

E-mail address: magnus.gislason@strath.ac.uk 


\section{Abstract}

Aims

To create an anatomically accurate three-dimensional finite element model of the wrist, applying subject specific loading and quantifying the internal load transfer through the joint during maximal grip.

\section{Methods}

For three subjects, representing the anatomical variation at the wrist, loading on each digit was measured during a maximal grip strength test with simultaneous motion capture. Internal metacarpophalangeal joint load was calculated using a biomechanical model. High resolution MR scans were acquired to quantify bone geometry. Finite element analysis was performed, with ligaments and tendons added, to calculate internal load distribution.

\section{Results}

For maximal grip the thumb carried the highest load, average of $72.2 \pm 20.1 \mathrm{~N}$ in the neutral position. Results from the finite element model suggested that the highest regions of stress were located at the radial aspect of the carpus. Most of the load was transmitted through the radius, $87.5 \%$ opposed to $12.5 \%$ through the ulna with the wrist in a neutral position.

\section{Conclusions}

$A$ fully three-dimensional finite element analysis of the wrist using subject specific anatomy and loading conditions was performed. The study emphasises the importance of modelling a large ensemble of subjects in order to capture the spectrum of the load transfer through the wrist due to anatomical variation.

Keywords: Wrist biomechanics; Gripping force; Finite element analysis; wrist ligaments; load transmission 


\title{
A 3D finite element model of maximal grip loading in the human wrist
}

\author{
Magnús K. Gíslason ${ }^{1 *}$, David H. Nash ${ }^{1}$, Alexander Nicol ${ }^{1}$, Asimakis Kanellopoulos ${ }^{1}$, \\ Marc Bransby-Zachary², Tim Hems ${ }^{3}$, Barrie Condon ${ }^{2}$, Benedict Stansfield ${ }^{4}$,
}

${ }^{1}$ Bioengineering Unit, Wolfson Building, University of Strathclyde, 106 Rottenrow, Glasgow, G4 0NW, UK

${ }^{2}$ Southern General Hospital, Glasgow, UK

${ }^{3}$ Victoria Royal Infirmary, Glasgow, UK

${ }^{4}$ Glasgow Caledonian University, Glasgow, UK

\section{Introduction}

The wrist is an anatomically complex joint. It is composed of 8 carpal bones assembled in a two row structure. In the proximal row are the (from radial to ulnar) scaphoid, lunate, triquetrum and pisiform. The pisiform is a sesamoid bone and plays no part in the overall load transfer. The distal row comprises of the (from radial to ulnar) trapezium, trapezoid, capitate and hamate [1]. Bone and ligamentous geometry are illustrated in Figure 1. There has been considerable debate about how load is transmitted through the joint over the last few decades. In 1981 some of the first wrist cadaveric measurements were carried out by Palmer and Werner [2] who used a load cell in order to establish the load transfer ratio between the radius and ulna. Other cadaveric studies followed in order to quantify the load transfer characteristics of the wrist. In 1987 and 1988, Viegas et al 
and Tencer et al [3-5] respectively performed cadaveric experiments using pressure sensitive films, placed at the articulating surface of the carpal bones in order to measure the contact pressures. The results from these cadaveric studies have shed light on how the wrist responds under loading, but concerns can be raised about the measuring procedures. Cadaveric measurements are difficult to perform. The wrist is a very delicate joint and by performing an invasive measurement it is possible that the researcher could be perturbing the joint as the dissection is carried out. Another issue with cadaveric studies is that after the specimen has been dissected and set up for experimental work, it is not possible to redo the experiment with modified parameters. This, along with difficulty in obtaining cadaveric specimens, makes these experiments time consuming and expensive to perform. Several theoretical models [6,7] of the wrist exist. These have been developed mostly by creating a Rigid Body Spring Model (RBSM) to calculate the force transmission and displacement between multiple non deformable bodies using a series of springs with known stiffness. The geometry of the wrist makes such theoretical models difficult to create. Finite element models of the wrist have been created, but most have focussed on a particular sub region of the joint, in particular the interaction between the radius, scaphoid and the lunate, not representing the whole joint. Exceptions to this include the work of Carrigan et al. in 2003 [8] who developed a three-dimensional FE analysis of the carpus (without metacarpals). In this work the bones were modelled as 'hollow' cortical shells with only a small number of the ligaments included. To obtain convergence of this model it was necessary to constrain each carpal bone with a system of non-physiological constraints. None of the models proposed in the literature used physiologically realistic loading systems; theoretical or arbitrary loads were applied. 
The aim of the current study was to develop a fully-representative three-dimensional finite element model of the entire wrist joint in order to study the transmission of force through the normal carpus during a maximal grip activity. A major consideration was the use of experimental biomechanical data which were obtained to provide 'real' boundary conditions for the model.

\section{Methods}

\subsection{Subjects}

The wrists of three subjects were studied. The three subjects ( 2 females and 1 male who all were young and healthy with average age of 26.3, ranging from 24-28 years) were selected from a group of 10 subjects who had MRI scans of the wrist. The subjects were selected to represent a range of wrist geometrical configurations, representing a range of wrist types proposed by Craigen and Stanley in 1995 [9] who suggested that the kinematics of the carpal bones varied depending on the rotational behaviour of the scaphoid.

\subsection{Activity}

The subjects performed a maximal strength grip with one finger on each force transducer

(Figure 2). This was performed in the neutral, radially deviated and ulnarly deviated positions. 


\subsection{Anatomical data collection}

The subjects were taken for an MRI scan at the Southern General Hospital in Glasgow. The subjects were scanned with their hands in three positions; neutral, radial deviation and ulnar deviation. The in-plane resolution of the MRI scan was $230 \times 230 \mu \mathrm{m}$ and the slice thickness was $700 \mu \mathrm{m}$. The image size was $512 \times 512$ pixels. The imaging consisted of 92 axially sliced scans ranging from the distal end of the radius and ulna to the proximal third of the metacarpals, a length in total of $63.7 \mathrm{~mm}$. The wrist of each subject was splinted while the imaging took place in order to keep the wrist as still as possible to minimise noise and maximise image quality.

\subsection{Biomechanical data collection}

Realistic external loading conditions must be applied to make the results of FE models valid. External loads were measured using individual finger transducers (Nano 25-E and Nano 17, ATI Industrial Automation Inc, USA) (Figure 2). The locations of the joint centres for each digit were determined from the position of skin mounted markers and from anatomical features, identified from static calibration trials. From the force transducer outputs, the inter-segmental loadings in terms of forces across the joints were calculated. Load was then distributed to the internal structures (tendons, ligaments and bones) at each joint using an inverse dynamic approach that used an optimisation criteria that minimised the maximum stress in any of the soft tissue structures as described by Fowler and Nicol $[10,11]$. The biomechanical model included all the tendons crossing the wrist that had attachment points distal to the metacarpals. Therefore 5 tendons were 
excluded, flexor carpi radialis, flexor carpi ulnaris, extensor carpi radialis longus, extensor carpi radialis brevis and extensor carpi ulnaris, as the attachment points of these tendons were at the proximal end of the metacarpals. These tendons were included in the FE model. This process used real external loading data and person specific anatomical data thus providing physiologically relevant loading information.

\subsection{Finite element model}

\subsubsection{Mesh generation}

The MRI scans were imported into Mimics software (Materialize, Belgium) where edge detection of the bones was carried out. By using the 'masking technique' all the bones and articulating cartilage were manually identified from each slice, thus creating a layer of contour surfaces in a plane perpendicular to the axis of the scan. Three-dimensional surface objects were created of each bone by combining the contours. Meshing was carried out using triangular elements on the surface objects using an automatic procedure. To remove surface roughness caused by the digitisation process, a smoothing function was applied which took each node point and changed its position in relation to the positions of the adjacent node points. The consequence of this procedure was a volume reduction within the bones. By recalculating the bone mask, based on the smoothed $3 \mathrm{D}$ object, it was possible to return to the original scans and compensate for the volume reduction. This became an iterative process, which was carried out until the volume change from 'before' to 'after' applying the smoothing function became negligible. The

triangular mesh was automatically and manually adjusted. Surface element density 
ranged from 1.91 elements $/ \mathrm{mm}^{2}-3.93$ elements $/ \mathrm{mm}^{2}$ (average of 2.73 elements $/ \mathrm{mm}^{2}$ ). Various indicators were used to identify badly shaped triangles such as the area ratio, the skewness defined as the ratio between the triangle and an equilateral triangle with the same ascribed circle and the equi-angle skewness which was defined as

$$
\min \left(\frac{\alpha}{60} ; \frac{180-\beta}{180-60}\right)
$$

where $\alpha$ was the smallest angle of the triangle and $\beta$ was the largest angle of the triangle. Ideally all the ratios would have been 1 , where that would have described an equilateral triangle. In practice it is difficult to create a mesh using only equilateral triangles. The minimum value used for the meshing was 0.4 . This value was considered to be acceptable based on the characterisation of equi-angle skew factor of Rábai and Vad in 2005 [12].

The mesh was refined by manually deleting and creating triangles. A histogram was plotted of the mesh quality indicators and visually evaluated before accepting the surface mesh quality. The meshes were then exported from Mimics and imported into Abaqus (v. 6.6-1, Simulia, USA) where volume elements were created from the surface elements. The volume elements were 10 node solid tetrahedral elements (C3D10). For this method of implementation the volume element shape is determined by the surface element shape which could possibly lead to the creation of distorted elements [13]. With modern mesh generators, the robustness of the algorithm minimizes the risk of that happening. However, the following checks were carried out on the mesh to make sure the elements were of sufficient quality: Shape factor, minimum face angle, maximum face angle, aspect ratio. If an element showed signs of distortion, the surface mesh was altered and the process repeated until all the volumetric elements were of sufficient quality. 


\subsubsection{Bone material property assignment}

It was not possible to derive the stiffness of each element of bone based on the greyscale value from the MR images. Areas of different stiffness were visually identified from the scans, within Mimics. This was performed by eroding the mask containing the bone and the articulating cartilage, thus creating areas of different stiffness using Boolean operators. These areas represented the hard cortical shell, the soft cancellous bone, two transition regions bridging the stiffness values between the hard cortical shell and the soft cancellous region, and finally, the cartilage. The stiffness values can be seen in Table 1 . The values for cortical bone were taken from Rho et al. [14] and for cancellous bone from Kabel et al. [15]. The values for the two transition stiffness regions were estimated and followed a modulus-density power curve proposed by various empirical studies [e.g. $16]$.

\subsubsection{Model assembly}

The volumetric elements with the bone material property definitions were imported into Abaqus where the assembly took place. The ligaments and tendons were modelled as non linear spring elements. Material properties of the soft tissues were taken from the literature. Material testing data were found for 26 ligaments [17-29]. All major ligaments were included in the model. The attachment points were estimated from various anatomical studies $[30,1]$. There is currently no non-invasive method of establishing the 
ligament origins and insertions in a live subject. The origins and insertion points of ligaments are diverse in nature, with multiple fibre attachment points. To make the modelling of ligaments possible they were applied as single elements, but with a distributed origin and insertion achieved by linking adjacent node points.

For ligaments that did not have published material parameters, it was assumed that the properties of the neighbouring ligaments would apply. An exception to this was for the transverse metacarpal ligaments which were modelled as being stiff to prevent large relative movements between the metacarpals.

The non linear curves of the ligaments were generated using the following points:

- Zero stress $=$ zero strain

- Non-linear 'toe' region up to $15 \%$ of max strain, called $s_{r e f}$.

- Linear curve from $15 \%$ max strain to max strain with same slope at the boundary between the linear and nonlinear regions, based on the formula below derived from results of Logan and Nowak [25].

$$
F=\left\{\begin{array}{c}
\frac{a}{2 a_{r e f}} x^{2}, \quad 0 \leq x \leq s_{\text {ref }} \\
a x+b, x \rightarrow s_{\text {ref }}
\end{array}\right.
$$

Where $a, b$ are constants, $F$ the force and $x$ the strain.

All tendons in the fingers that run across the wrist were modelled in the biomechanical model for calculating the MCP joint loading. In addition the intrinsic tendons were modelled in the FE simulation. The contributions from the wrist flexors (flexor carpi radialis and flexor carpi ulnaris) and extensors (extensor carpi radialis longus and brevis and extensor carpi ulnaris) were included separately in the model by non-linear axial 
constraints, with moment arms taken from Horii et al. [31] for the joint position defined by the MRI scan. Thus all tendon loading was included in the load distribution calculations. The material properties of the tendons were taken from a material study of the wrist tendons carried out at the University of Strathclyde [32]. The ligaments and tendons were modelled as non linear to improve physiological relevance.

There were clear limitations in the ability of the ligament and tendon structures modelled to offer load resistance perpendicular to their lines of action. This is a simplification of the action of these structures. The lines of action of the structures were examined during the simulations and only minor intrusions of them into the bone were observed. It was therefore concluded that using this representation of the ligaments and tendons was justifiable as it provided a physiological representation of the main resistance (longitudinal loading) and minimal compromise of the model integrity.

The number of elements in each assembly of bones in the wrist model ranged from 172,413 to 274,261 (average 228,771) with element density ranging from 6.58 to 9.07 elements $/ \mathrm{mm}^{3}$ (average of 7.74) per bone for all the models.

The models were solved using Abaqus explicit solver v6.6-1 and run on a 4 dual $2 \mathrm{GHz}$ processor cluster with 4Gb of RAM. The analysis took 25-30 hours of CPU time for each of the models. The 3 models were run using the explicit solver with a total simulated time of $1 \mathrm{~ms}$. 


\subsubsection{Boundary conditions}

The action of the external applied loads and the digital extrinsic muscles on the wrist joint were accounted for by the joint contact loads defined at the metacarpals. These three-dimensional metacarpal forces were applied over the distal surface of each metacarpal as a set of boundary conditions.

The proximal surface of the radius and ulnar were constrained by allowing no displacement or rotation in any direction. The proximal ends of the radius and ulnar were assumed to be completely rigid.

\subsubsection{Contact modelling}

The contact modelling using the explicit solver, assumed that all the exterior elements were in contact. There was therefore no need for a predefined notion of where the contact surfaces lay. The bones were translated until they were touching their adjacent bone surface.

A surface-to-surface contact was established between the bones using the 'hard contact' algorithm based on

$$
\begin{aligned}
& p=0, \text { for } h<0 \\
& h=0, \text { for } p>0
\end{aligned}
$$


where $p$ was the contact pressure and $h$ was the overclosure between the surfaces. The contact modelling was implemented in Abaqus code. An additional tangential component was established. The tangential component was modelled as friction based on the classical Coulomb friction model where

$$
\tau=\mu p
$$

Where $\tau$ is the shear stress, $\mu$ is the friction coefficient and $p$ is the contact pressure. The friction modelling assumed no upper boundaries on the shear stress, thus allowing no relative motion as long as the surfaces were in contact. Ideally the carpal bones would have had frictionless contact, but applying a frictionless model resulted in divergence. These adjustments were important for the convergence of the modelling.

\section{Results}

\subsection{External loading}

The joint coordinate systems used are illustrated in Figure 3. Table 2 details the overall external loading effect at the digit tips in the transducer coordinate system. The forces applied normal to the contact surface of the transducers were highest for the thumb, averaging at $72.2 \mathrm{~N}$. The average normal forces for the index, middle, ring and little finger were $20 \mathrm{~N}, 25 \mathrm{~N}, 24 \mathrm{~N}$ and $11 \mathrm{~N}$ respectively. Table 3 details the external force effects at the metacarpophalangeal joint in the coordinate system of the metacarpals as 
illustrated in Figure 3. The proximal force components for digits 2-5 were higher as a percentage of the resultant force than the corresponding proximal force component for the thumb. Higher percentage was seen in the dorsal component of the thumb than for the other four digits. This was in agreement with the positioning of the fingers on the gripping tool, where the distal interphalangeal joint angles were higher in digits 2-5 than the thumb, resulting in the load being applied predominantly in the dorsal direction in the thumb phalangeal coordinate system.

\subsection{Metacarpophalangeal joint loads}

It can be seen in Table 4 that the proximal component of the metacarpophalangeal joint contact force $(F y)$ was the highest, as a consequence of the tendon forces pulling the bones together. From Table 4 it can be seen how the values differed from the external loading, with the highest force-component acting proximally due to the contribution from the tendons which were incorporated into the biomechanical model.

The resultant forces acting on the metacarpals were calculated by taking the quadratic sum of the three force components for all the digits as:

$\begin{array}{lll}\text { Neutral position: } & 1836 \mathrm{~N}, 1232 \mathrm{~N}, 1350 \mathrm{~N} & \text { for subjects } 1,2 \text { and } 3 \text { respectively } \\ \text { Radial deviation: } & 1568 \mathrm{~N}, 1231 \mathrm{~N}, 976 \mathrm{~N} & \text { for subjects } 1,2 \text { and } 3 \text { respectively } \\ \text { Ulnar deviation: } & 1453 \mathrm{~N}, 1004 \mathrm{~N}, 949 \mathrm{~N} & \text { for subjects } 1,2 \text { and } 3 \text { respectively }\end{array}$




\subsection{Bone stress distribution}

Surface stress contour plots can be seen in Figures 4a-c, for subjects 1, 2, and 3

respectively. It was observed that the stress distribution through the carpus was different between subjects. Subject 1 showed high stresses down the radial aspect of the carpus, in particular through the trapezium, trapezoid and the scaphoid. For subject 3 it could be seen that the palmar side of the capitate was highly loaded which subsequently was directed to the lunate. Subject 2 showed more concentrated stresses in the radius which relieved the loading on the ulna. The stress density of the scaphoid is visibly the highest for subject 1 (Figure 4a). By averaging the stress values for each of the 4 integration points in the 10-node tetrahedral elements it was found that between $91.9 \%$ and $96.9 \%$ of the elements had von Mises stresses below $50 \mathrm{MPa}$, with the vast majority of elements below 20MPa with the wrist in a neutral position (Figure 5). The corresponding numbers for radial deviation were $96.4 \%$ and $99.0 \%$ and for ulnar deviation $94.5 \%$ and $97.2 \%$. This was in agreement with the fact that the input loads were lower for the radially and ulnarly deviated positions than for the neutral position. High stress intensity regions could be seen at the insertion/origin points of the ligaments and in the surrounding elements due to the coupling of the node points. These stresses were though highly localized.

The strain values calculated for the cartilage on the radius were on average (standard deviation) $\varepsilon=-14.4 \%$ (27.0\%) for the three subjects in a neutral position. The corresponding value for the cortical bone was $\varepsilon=-0.13 \%(0.56 \%)$ and for the cancellous bone $\varepsilon=-0.81 \%(0.59 \%)$. The stresses in the bones were higher in the cortical shell than 
the cancellous region. Results in ulnar and radial deviation showed similar stress distribution as in the neutral position with the majority of the loading travelling through the radial aspect of the carpus.

\subsection{Ligament forces}

Table 5 shows the forces acting in a selected set of ligaments. The forces in the radiotriquetral ligament ranged from $12.4 \mathrm{~N}$ to $74.4 \mathrm{~N}$ for all the models and were highest in the neutral position averaging to $37.2 \mathrm{~N}$, opposed to average values of $20.4 \mathrm{~N}$ and 19.7 $\mathrm{N}$ in radial and ulnar deviation respectively. Other ligaments that showed high activity were the scaphotrapezoid band with average tension force of $147.3 \mathrm{~N}$ in a neutral position, $152.1 \mathrm{~N}$ in radial deviation and $155.3 \mathrm{~N}$ in ulnar deviation. The scaphotrapezium band averaged at $26.0 \mathrm{~N}$ in neutral position, $109.7 \mathrm{~N}$ in radial deviation and 53.6 $\mathrm{N}$ in ulnar deviation. Less activity was seen in the scapholunate ligament which averaged at $13.5 \mathrm{~N}$ in neutral position, $1.0 \mathrm{~N}$ in radial deviation and 8.2 in ulnar deviation.

\subsection{Forearm bone force transmission}

Resultant reaction forces at the proximal end of the radius and ulna were calculated and the load transfer ratio between the two bones estimated. Table 6 shows the load ratio between the radius and ulna. Results showed that the load travelling through the radius varied depending on subject and position. From Table 6 it can be seen that the percentage 
values ranged from $78.7 \%$ to $92.8 \%$ with the wrist in a neutral position. On average more force was transmitted through the ulna during radial and ulnar deviation. This was confirmed through a cadaveric study carried out at the University of Strathclyde, Glasgow. Strain gauges were placed on the scaphoid, lunate, radius and ulna and strain measurements taken with the wrist undergoing similar loading conditions as described above. The results showed that on average $68 \%$ was transmitted through the radius and $32 \%$ through the ulna [33].

\section{Discussion}

\subsection{Loading conditions}

The joint contact values were reflective of the fact that the subjects were able to produce the highest gripping force in neutral position and the lowest in ulnar deviation. The joint contact forces ranged from 1.5-2.0 bodyweight between the subjects which might be considered to be extremely high for a joint which is not a body weight bearing joint. These force values were for maximal grip loading and so represent the maximum forces that the subjects could apply. It would therefore be expected that the internal loading be high in comparison with maximal possible (i.e. failure) loads. Subjects were only required to maintain grip force of a few seconds duration. The joint contact force values are thought to represent upper boundaries of the physiological loading. Other finite element studies $[8,34]$ have applied lower forces acting on the carpal bones. 


\subsection{Model construction}

It was not possible to use automatic edge detecting procedures for automatic creation of the models due to insufficient resolution of the MR scans. It was therefore necessary to carry out edge detection manually. Great care was taken to recreate the anatomy as accurately as possible. In order to check consistency of the geometrical construction, the volume of the capitate bone was compared for the subjects for each position. It was found that the deviation from the average value ranged between $3.6 \%-6.5 \%$ for subject $1,0.2 \%$ $2.9 \%$ for subject 2 and $0.8 \%-4.8 \%$ for subject 3 which showed that the repeatability of the geometrical modelling was high. It was assumed therefore that the models represented all of the bones with high geometrical accuracy.

\subsection{Contact modelling}

The contact between the bones was modelled so that once the bones had established contact it was not possible for the bones to separate. This was necessary in order to improve the stability of the carpus, particularly in the dorsal/palmar directions under the influence of the shear forces.

\subsection{Requirements for model convergence}

The stability of the carpus is dependent on contributions from soft tissue structures, mainly the ligaments and tendons. As the ligaments were modelled using onedimensional springs, the transverse stability generated by them in the wrist was not fully included in the model. To overcome this limitation it was necessary to add constraints on the relative motion of the metacarpals by using stiff ligament representations once contact 
had been established. Modelling the transverse metacarpal ligaments as stiff did alter the internal load distribution. However, this effect was predominantly in a medio-lateral direction and the effects along the main loading axis were minimal.

To enhance convergence it was also necessary to prevent separation of the bones once contact had been made. Attempts were made to use frictionless behaviour at the contacts but this resulted in divergence where the bones became separated from each other. The precautions put in place prevented dorsal/palmar instability and allowed model solution.

\subsection{Stress of the carpal bones}

Linearly elastic bone and cartilage material properties were used. The loading conditions explored were of short duration and effectively static in the 'hold' phase of maximal grip

loading. It was therefore considered reasonable to ignore viscoeleastic effects. There has been no attempt to include an exploration of the nonlinear aspect of bone and cartilage behaviour. Inhomegeneity and anisotropy within the materials were not included. Evaluation of inhomegeneity would to some extent be possible using statistical methods with materials described using a distribution of properties. Anisotropic effects could be included based on definitions of the radial and transverse directions within bones.

Exploration of the effects of these material properties on the stress distribution would clearly be desirable, although this would add considerably to the computational solution time and introduce further assumptions as accurate, local material property characterisation is not possible for the whole wrist. 
From the stress distribution of the model it could be seen how the stress varied over the carpal bones. The findings show similar results to the ones of Ulrich et al [34] who reported that $93 \%$ of the elements were stressed between 0 and $30 \mathrm{MPa}$ (von Mises stresses) in a 3-bone model consisting of the radius, scaphoid and lunate. The corresponding values based on the current model were $83.6 \%, 92.8 \%$ and $87.3 \%$ for subjects 1,2 and 3 respectively. The loading conditions of Ulrich et al's model consisted of a total load of $1000 \mathrm{~N}$ distributed between the scaphoid and lunate whereas the loading conditions applied for the present model ranged from $1232 \mathrm{~N}$ to $1835 \mathrm{~N}$, so that the input loads were higher, resulting in higher stress values. Another reason for the higher percentage of elements loaded beyond $30 \mathrm{MPa}$ was the fact that the ligament attachment points showed high peak stresses which were distributed in the neighbouring elements but died down rapidly after that. The application points of the loading onto the model also exhibited high stress. These stress values were not thought to be representative of physiological conditions.

The average strain values calculated for the cartilage, cancellous and cortical bone were all within the physiological range. Bosisio et al. [35] presented ultimate strain values of the cortical bone in the radius to be $\varepsilon_{\mathrm{u}}=1.5 \pm 0.1 \%$ and the yield strain $\varepsilon_{\mathrm{y}}=0.9 \pm 0.2 \%$ so the strains presented in the current model were below the failure criteria. The cartilage underwent higher values of strain and averaged at $\varepsilon=-14.4 \%$ over the whole radiocarpal joint. The failure strain value of cartilage under compression has been published by Kerin et al. [36] to be $30 \%$. For the 3 subjects in a neutral position it was found that on average $13.5 \%$ of the cartilage elements exceeded compressive strains of $30 \%$, which could 
indicate that some of the cartilage could be damaged under such loading conditions or that local imperfections in the model geometry caused unphysiological strain values.

\subsection{Ligaments}

The ligamentous contribution could be seen as localized stress increases but died out rapidly and would have had minimal effect on the overall stress distribution at the joints. This was due to the point to point connection of the ligaments in the model and was most clearly seen in the transverse metacarpal ligaments which were modelled as stiff and thus did not allow any extension. This was necessary as there were no other factors contributing to the stabilization of the metacarpals within the model.

The palmar ligaments were in general more load bearing than the dorsal ligaments and the results are in agreement with the theoretical model presented by Garcia-Elias in 1997 [37], where it was proposed that for gripping, the main stabilizing ligamentous structures for the carpus are the scapho-trapezium-trapezoid ligaments, the scapho-triquetral ligament and the radiotriquetral ligament. The radiotriquetral ligament showed high load for all the subjects with the wrist in all positions. Less load was seen through the scaphotriquetrum than expected due to Garcia-Elias' theory, but this transverse stability was compensated elsewhere in the models, such as in the capitotrapezoid, capohamate and hamotriquetrum ligaments.

\subsection{Validation}

Validation of finite element model results is critical to provide confidence that the calculated load distributions are reasonable.

Deleted: Cadaveric study results were available and these demonstrated general agreement with the outputs of this finite element study [33]. 
The relevance of subject specific anatomy to the outcomes of load distribution has been demonstrated in the current study. It is therefore difficult to use the evidence from a cadaveric study [33] on a wrist with a different anatomical configuration to directly validate the current work. However, the cadaveric study results that were available demonstrated general agreement with the outputs of this finite element study. The authors are not aware of any reliable techniques that might be applied to subjects in vivo to assess ligament loading and bone stress distributions without disrupting natural load transfer characteristics. Further cadaveric work is desirable as maximal grip loading with physiological load application has not been studied extensively.

\section{Conclusions}

The wrist model presented here offers major steps forward in the long process of creating a physically representative numerical simulation of the wrist joint. This study demonstrated how a geometrically accurate finite element model of a complex joint can be constructed in a time efficient manner in order to be able to model anatomical differences between subjects and to identify them as a part of a larger ensemble. This gives the possibility of predicting load distributions following interventions in the wrist to inform surgical planning. The differences observed in the load distribution in the wrist

joints studied, emphasises the need to move away from the idea of an 'average' standard model and to capture individual specific information. 


\section{Acknowledgements}

The authors wish to thank Dr. N K Fowler for her input in the modelling, our subjects and the Furlong Foundation and the Arthritic Research Campaign who funded this work.

\section{References}

1. McGrouther, D.A. and Higgins, P.O., Interactive Hand - Anatomy CD, Primal Pictures, v.1.0

2. Palmer, A.K. and Werner, F.W., Biomechanics of the Distal Radioulnar Joint, Clin. Orthop. Rel. Res, 1984, 187, 26-35.

3. Viegas, S.F., Tencer A.F., Cantrell, J., Chang, M., Clegg, P., Hicks, C., O’Meara, C., and Williamson, J.B., Load Transfer Characteristics of the Wrist: Part 1, the Normal Joint, American Society for Surgery of the Hand, 1987, 12A(6), 971-978.

4. Viegas, S.F., Tencer, A.F., Cantrell, J., Chang, M., Clegg, P., Hicks, C., O’Meara, C., and Williamson, J.B., Load Transfer Characteristics of the Wrist: Part 2, Perilunate Instability, American Society for Surgery of the Hand, $1987,12 \mathrm{~A}(6), 978-985$.

5. Tencer, A.F., Viegas, S.F., Cantrell, J., Chang, M., Clegg, P., Hicks, C., O’Meara, C., and Williamson, J.B., Pressure distribution in the Wrist Joint, Journal of Orthopaedic Research, 1988, 6(4), 509-517.

6. Schuind, F., Cooney, W.P., Linscheid, R.L., An K.N., and Chao, E.Y.S., Force and Pressure Transmission Through the Normal Wrist: A Theoretical Two- 
Dimensional Study in the Posteroanterior Plane, Journal of Biomechanics, $1995,28(5), 587-601$.

7. Nedoma J., Klézl Z., Fousek J., Kestránek Z., Stehlík J, 2003, Numerical Simulation of Some Biomechanical Problems, Mathematics and Computers in Simulation, 61, 283-295

8. Carrigan, S.D., Whiteside, R.A., Pichora, D.R., and Small, C.F. Developement of a Three Dimensional Finite Element Model for Carpal Load Transmission in a Static Neutral Posture, Annals of Biomedical Engineering, 2003, 31, 718725.

9. Craigen, M.A.C. and Stanley, J.K., Wrist Kinematics: Row, Column or Both, Journal of Hand Surgery (British and European Volume), 1995, 20B(2), 165170.

10. Fowler, N.K. and Nicol, A.C., Interphalangeal Joint and Tendon Forces: Normal Model and Biomechanical Consequences of Surgical Reconstruction, Journal of Biomechanics, 2000, 33, 1055-1062.

11. Fowler, N.K. and Nicol A.C., Functional and Biomechanical Assessment of the Normal and Rheumatoid Hand, Clinical Biomechanics, 2001, 16(8), 660-666.

12. Rabai, G. and Vad, J., Validation of a Computational Fluid Dynamics Method to be Applied to Linear Cascades of Twisted-Swept Blades, Periodica Polytechnica Ser. Mech. Eng, 2005, 49(2), 163-180

13. Viceconti, M., Bellingeri L., Cristofolini, L., and Toni, A., A Comparative Study on Different Methods of Automatic Mesh Generation of Human Femurs, Medical Engineering and Physics, 1998, 20, 1-10. 
14. Rho, J.Y., Tsui, T.Y., and Pharr, G.M., Elastic properties of human cortical and trabecular lamellar bone measured by nanoindentation, Biomaterials, 1997, 18(20), 1325-1330.

15. Kabel, J., Odaard, A., van Rietbergen, B. and Huiskes, R., Connectivity and the elastic properties of cancellous bone, Bone, 1999, 24(2), 115-120.

16. Morgan, E.F., Bayraktar, H.H., Keaveny, T.M., Trabecular bone modulusdensity relationships depend on anatomic site. Journal of Biomechanics 36, 2003: 897-904

17. Berger, R.A., Imeada, T., Berglund, L., and An, K.N., Constraint and material properties of the subregions of the scapholunate interosseous ligament., J Hand Surg [Am], 1999,24(5), 953-62.

18. Bettinger, P.C., Linscheid, R.L., Berger, R.A., Cooney, W.P., and An, K.N., An Anatomic Study of the Stabilizing Ligaments of the Trapezium and Trapeziometacarpal Joint, Journal of Hand Surgery,1999, 24(4), 786-798.

19. Bettinger, P.C., Smutz, W.P., Linscheid, R.L., Cooney, W.P., and An, K.N., Material Properties of the Trapezial and Trapeziometacarpal ligaments, Journal of Hand Surgery, 2000, 25A, 1085-1095.

20. Cuénod, P., Charriére, E., and Papaloïzos, M. Y., A Mechanical Comparison of Bone-Ligament-Bone Autografts From theWrist for Replacement of the Scapholunate Ligament, Journal of Hand Surgery, 2002, 27A, 985-990.

21. Harvey, E. and Hanel, D., Bone-Ligament-Bone Reconstruction for Scapholunate Disruption, Techniques in Hand and Upper Extremety Surgery, $2002,6(1), 2-5$. 
22. Harvey, EJ, Hanel, D., Knight, J.B., and Tencer, A.F., Autograft replacements for the scapholunate ligament: a biomechanical comparison of hand-based autografts., J. Hand Surg [Am],1999, 24(5), 963-7.

23. Hofstede, D.J., Ritt, M.J., and Bos, K.E., Tarsal autografts for reconstruction of the scapholunate interosseous ligament: a biomechanical study,1999, J Hand Surg [Am], 24(5), 968-76

24. Johnston, J.D., Small, C.F., Bouxsein, M.L., and Pichora, D.R., Mechanical Properties of the Scapholunate Ligament Correlate with Bone Mineral Density Measurements of the Hand, Journal of Orthopaedic Research, 2004, 22, 867871.

25. Logan, S.E. and Nowak, M.D., Distinguishing Biomechanical Properties and Intrinsic and Extrinsic HumanWrist Ligaments, Journal of Biomechanical Engineering, 1991,113(1), 85-93.

26. Lutz, M., Haid, C., Steinlechner, M., Kathrein, A., Arora, R., Fritz1, D., Gabl, M., and Pechlaner, S., Scapholunate ligament reconstruction using a periosteal flap of the iliac crest: a biomechanical study, Archives of Orthopaedic and Trauma Surgery, 2004,124(4), 262-266.

27. Ritt, M.J., Berger, R.A., Bishop A.T., and An, K.N., The capitohamate ligaments. A comparison of biomechanical properties., J Hand Surg [Br], 1996, 21(4),451-454.

28. Svoboda, S.J., Eglseder Jr, W.A., and Belkoff, S.M., Autografts from the foot for reconstruction of the scapholunate interosseous ligament., J Hand Surg [Am], 1995, 20(6),980-5. 
29. Shin, S.S., Moore, D.C., McGovern, R.D., and Weiss, A.P., Scapholunate ligament reconstruction using a bone-retinaculum-bone autograft: a biomechanic and histologic study., J Hand Surg [Am], 1998, 23(2), 216-21.

30. Berger, R.A. and Garcia-Elias, M., Biomechanics of the Wrist Joint, SpringerVerlag, NY, 1991, Chapter: General Anatomy of the Wrist, 1-22.

31. Horii, E., An, K.N., and Linscheid, R.L., Excursion of Prime Wrist Tendons, Journal of Hand Surgery, 1993, 18A, 83-90.

32. Deakin, A. H., The Mechanical and Morphological Properties of Normal and Rheumatoid Human Forearm Tendons, 2006, Ph.D. thesis, University of Strathclyde.

33. Macleod, N.A., Nash D.H., Stansfield B.W., Bransby-Zachary M., Hems T.. Cadaveric Analysis of the Wrist and Forearm Load Distribution for Finite Element Validation, Proceedings of the 6th International Hand and Wrist Biomechanics Symposium, Tainan, Taiwan 2007, 11.

34. Ulrich, D., van Rietbergen, B., Laib, A., and Ruegsegger, P., Load Transfer Analysis of the Distal Radius From In-vivo High Resolution CT-imaging, Journal of Biomechanics, 1999, 32, 821-828.

35. Bosisio, M., Talmant M., Skalli, W., Laugier, P., and Mitton, D., Apparent Young's modulus of human radius using inverse finite-element method, Journal of Biomechanics 40 (2007) 2022-2028

36. Kerin, A.J., Wisnom, M.R., and Adams, M.A., The compressive strength of articular cartilage, Proceedings of the Institution of Mechanical Engineers, Part H: Journal of Engineering in Medicine,1998, 212(4), 273-280 
37. Garcia-Elias, M., Kinetic Analysis of Carpal Stability During Grip, Hand Clinics, 1997,13(1), 151-158. 


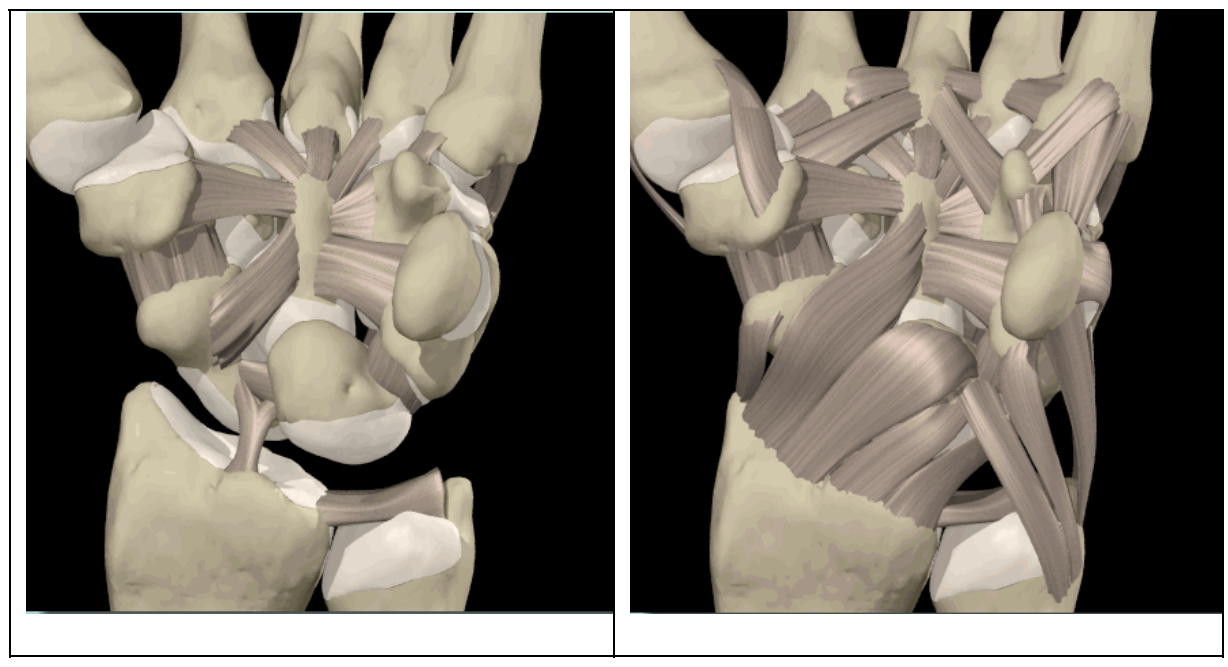

Figure 1: Palmar view of the wrist bones and the major ligaments

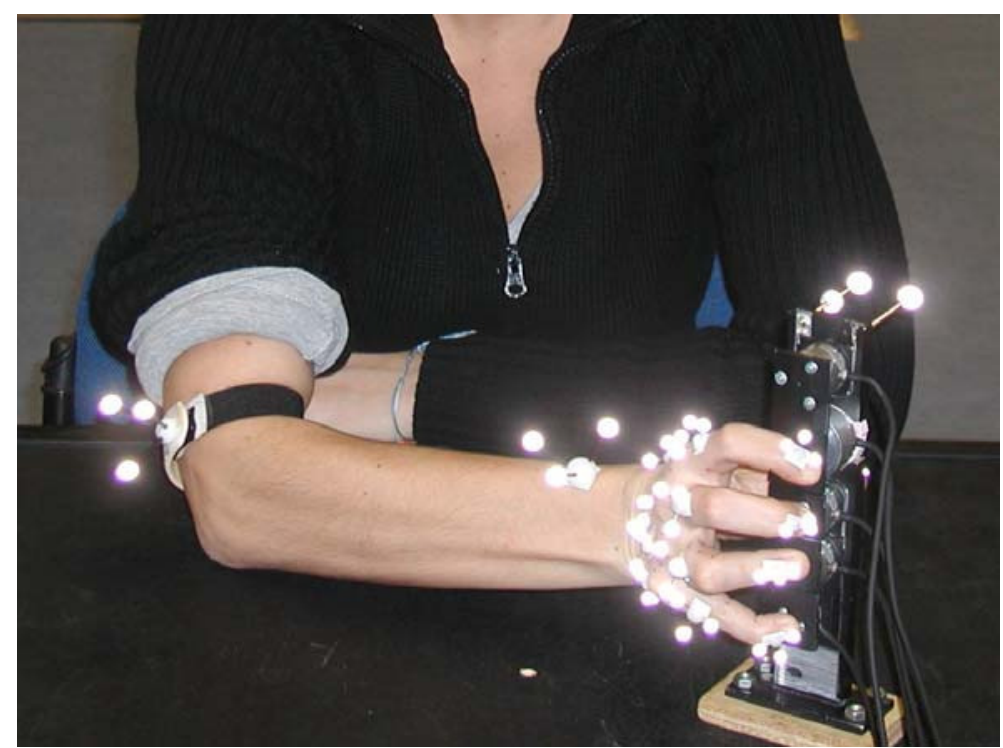

Figure 2. Subject performing the force experiment with the wrist in a functional neutral position. Motion capture markers are attached to the arm, hand and force measurement device. 


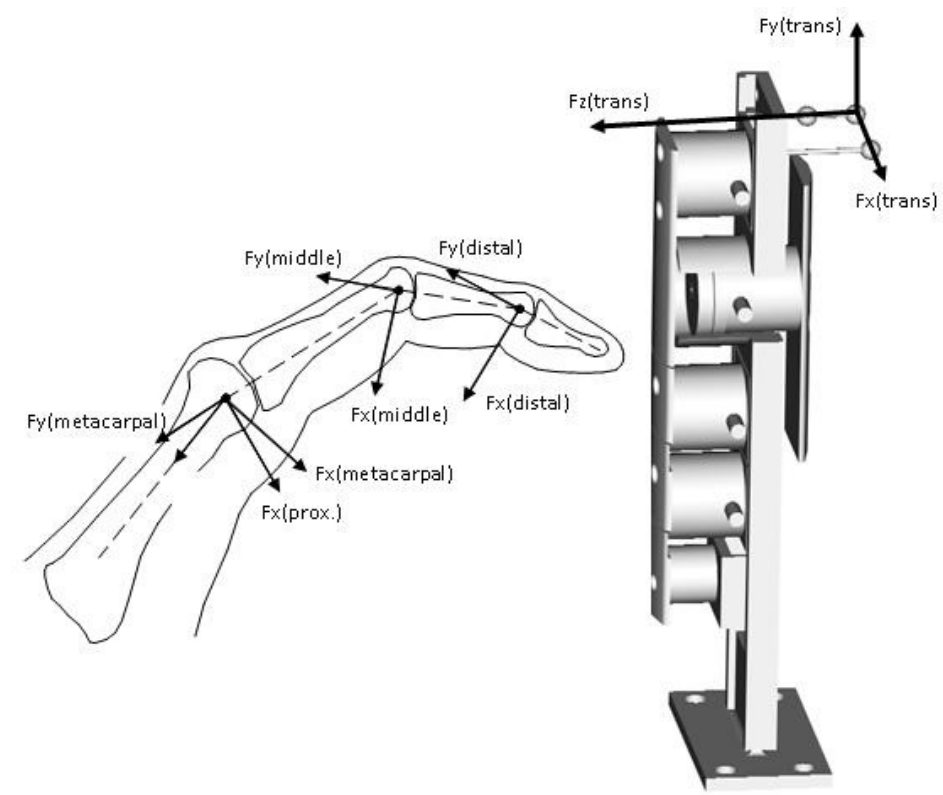

Figure 3: Coordinate systems used for the analysis of the gripping force and converting the externally measured loading into joint contact forces. Normal forces on the transducers were directed in the z-direction. In the phalanx coordinate system, the zdirection was radial for the right hand, $y$-direction was proximal and $x$-direction was palmar.

(trans $=$ transducer, distal, middle and proximal = phalangeal axes systems, metacarpal = metacarpal axis system) 


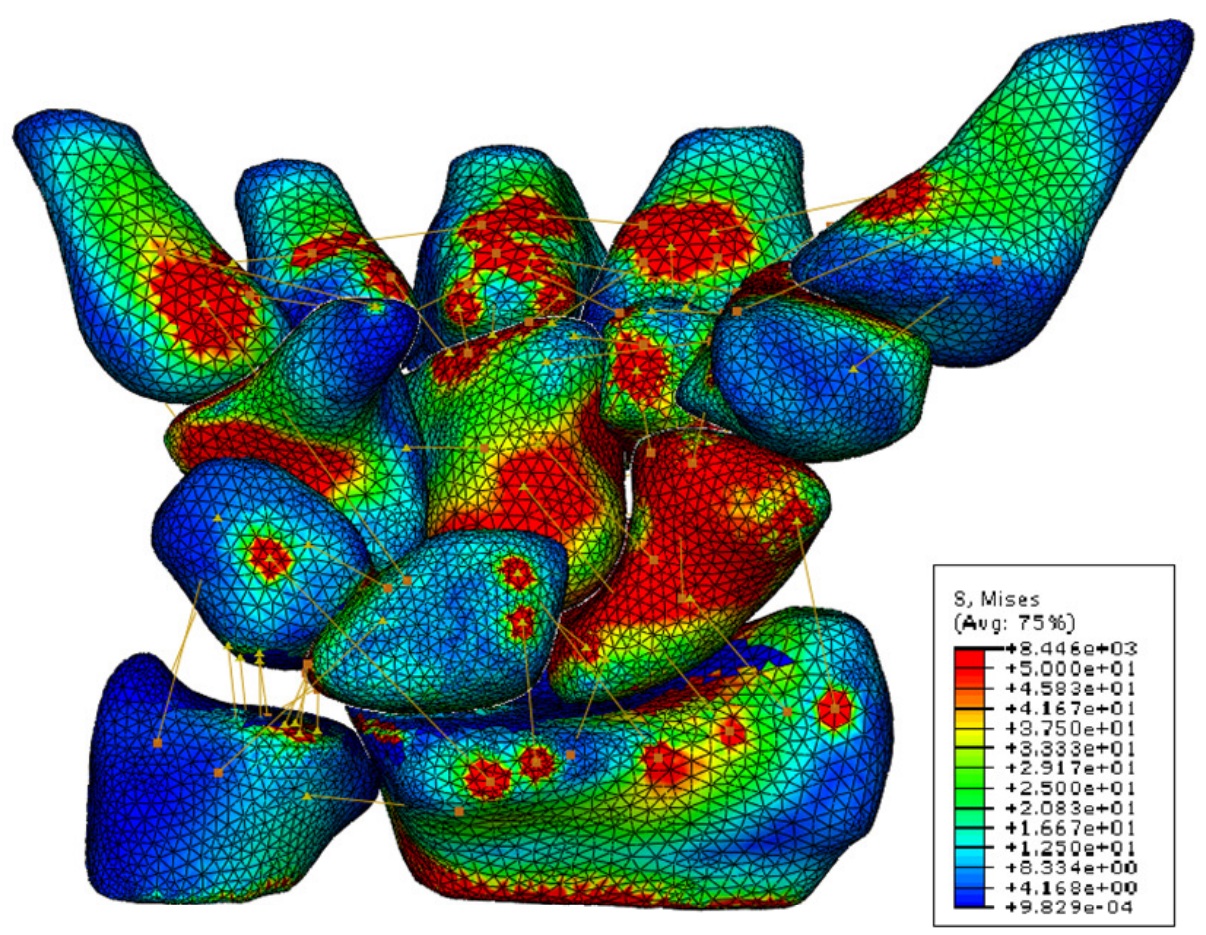

Figure 4a

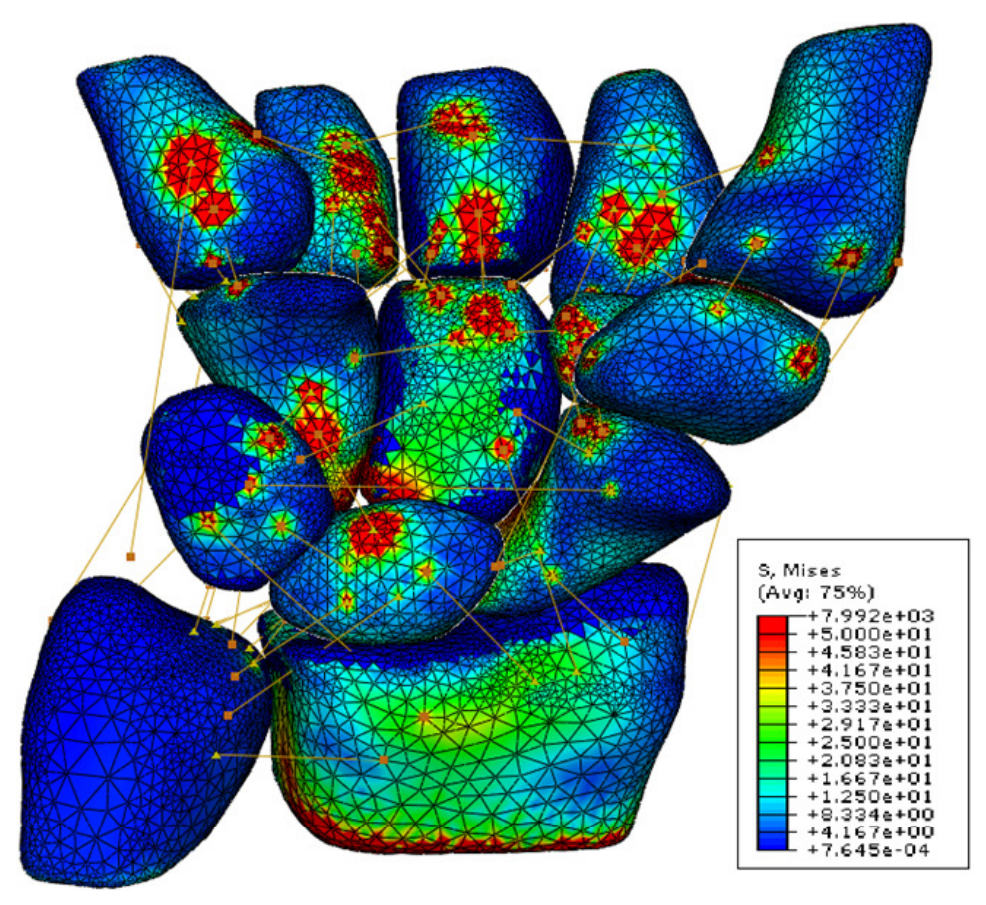


Figure 4b

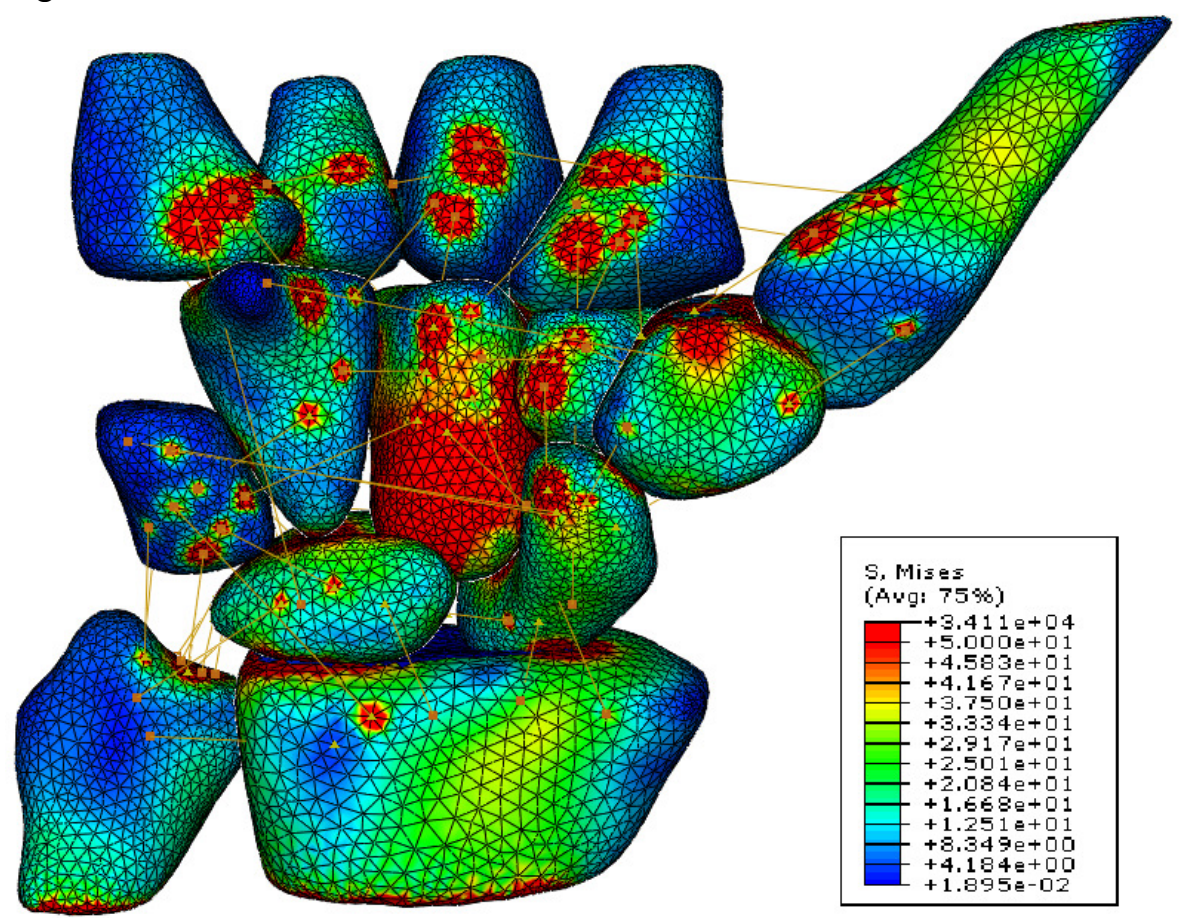

Figure 4c

Figure 4: von Mises (MPa) contour plots of the palmar aspect of the wrist joint for the 3 subjects (a, b, c subjects 1, 2, 3 respectively) with the wrist in the neutral position. Ligament elements are shown as yellow lines. 


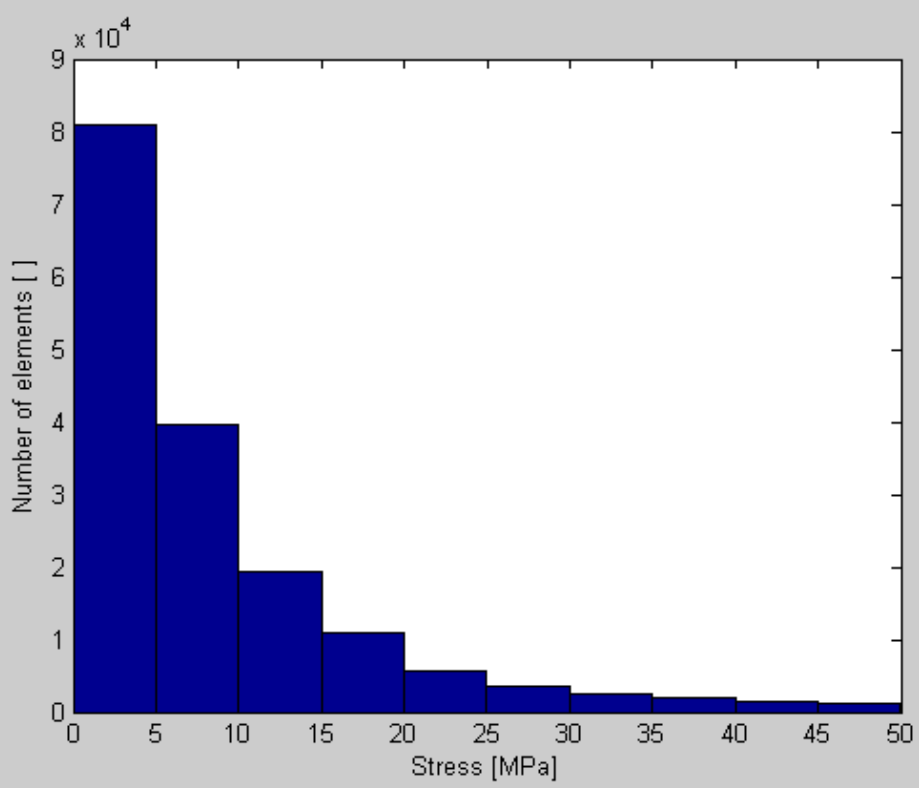

Figure 5: Histogram showing the distribution of the von Mises stress values in the model elements. Values above $50 \mathrm{MPa}$ are discarded from the histogram.

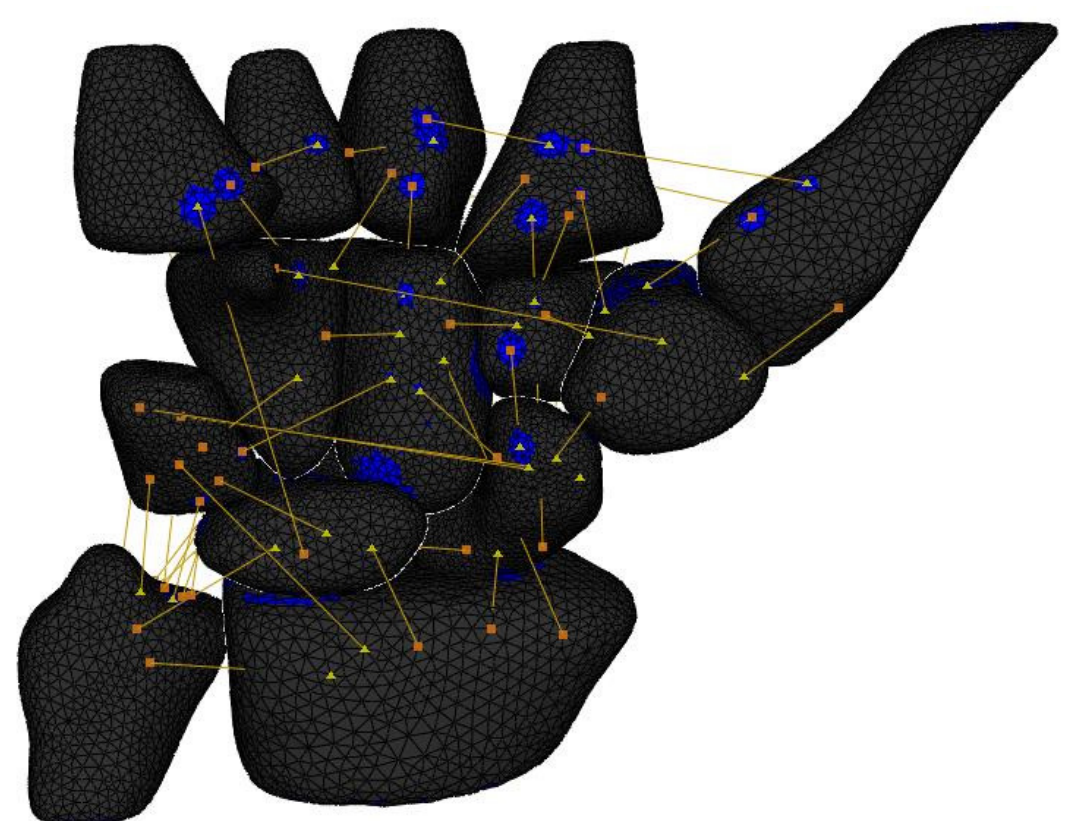

Figure 6: Positions of von Mises stresses exceeding $100 \mathrm{MPa}$. The blue elements represent elements stressed beyond $100 \mathrm{MPa}$. 


\begin{tabular}{|l|l|l|l|}
\hline & Young's modulus $[\mathrm{MPa}]$ & Poisson's ratio & Density $\left[\mathrm{g} / \mathrm{cm}^{3}\right]$ \\
\hline Cartilage & 10 & 0.4 & 1.1 \\
\hline Cancellous bone & 100 & 0.25 & 1.3 \\
\hline Subchondral bone soft & 1000 & 0.25 & 1.6 \\
\hline Subchondral bone hard & 10000 & 0.2 & 1.8 \\
\hline Cortical & 18000 & 0.2 & 2.0 \\
\hline
\end{tabular}

Table 1: Material properties used in the model

\begin{tabular}{|l|l|l|l|l|l|l|l|l|l|}
\hline & \multicolumn{3}{|c|}{ Subject 1 } & \multicolumn{3}{c|}{ Subject 2 } & \multicolumn{3}{c|}{ Subject 3 } \\
\hline Digit & Fx & Fy & Fz & Fx & Fy & Fz & Fx & Fy & Fz \\
\hline 1 & 1.2 & -12.3 & 99.0 & 10.2 & -12.5 & 67.2 & 0.5 & -6.0 & 50.6 \\
\hline 2 & 1.0 & -3.8 & -14.2 & -1.6 & -5.9 & -24.6 & -0.5 & -1.6 & -22.5 \\
\hline 3 & 8.3 & -11.0 & -41.0 & -3.2 & -0.4 & -20.8 & 0.2 & 0.3 & -13.5 \\
\hline 4 & -3.4 & -10.4 & -43.4 & -4.1 & 0.6 & -16.0 & -2.4 & 0.8 & -15.4 \\
\hline 5 & -2.5 & -2.7 & -16.7 & -0.8 & -2.8 & -12.3 & 1.0 & -1.1 & -6.8 \\
\hline
\end{tabular}

Table 2: External forces (Newtons) measured in the transducer coordinate system with the hand in a neutral position. The directions can be seen from Figure 3, with the Fz representing the normal force onto the force transducer, Fx and Fy represent the shear forces.

\begin{tabular}{|l|l|l|l|l|l|l|l|l|l|}
\hline & \multicolumn{3}{|c|}{ Subject 1 } & \multicolumn{3}{c|}{ Subject 2 } & \multicolumn{3}{c|}{ Subject 3 } \\
\hline Digit & Fx & Fy & Fz & Fx & Fy & Fz & Fx & Fy & Fz \\
\hline 1 & -98.0 & 11.9 & -13.7 & -51.0 & 18.0 & -43.1 & -46.9 & 5.6 & -19.0 \\
\hline 2 & -2.5 & -10.6 & -9.9 & -4.6 & -18.1 & -17.2 & -1.7 & -13.6 & -17.9 \\
\hline 3 & -26.7 & -32.6 & -9.7 & 2.0 & -15.9 & -13.7 & -1.0 & -9.8 & -9.2 \\
\hline 4 & -15.1 & -41.1 & -9.7 & -4.1 & -13.9 & -7.9 & -5.3 & -13.8 & -5.1 \\
\hline 5 & -5.3 & -16.2 & -0.2 & -4.8 & -11.3 & -2.6 & -4.0 & -5.3 & -2.3 \\
\hline
\end{tabular}

Table 3: External forces (Newtons) measured in the metacarpal coordinate system with the hand in a neutral position. The directions can be seen from Figure 3. +ve Fx force is palmarly directed, +ve Fy is directed proximally and +ve Fz is radially directed

\begin{tabular}{|l|l|l|l|l|l|l|l|l|l|}
\hline & \multicolumn{3}{|c|}{ Subject 1 } & \multicolumn{3}{c|}{ Subject 2 } & \multicolumn{3}{c|}{ Subject 3 } \\
\hline Digit & Fx & Fy & Fz & Fx & Fy & Fz & Fx & Fy & Fz \\
\hline 1 & 144.1 & -545.1 & -44.6 & 80.8 & -536.1 & -8.4 & 139.7 & -452.2 & -12.0 \\
\hline 2 & 253.2 & -270.7 & 141.8 & 84.1 & -294.2 & 10.5 & 110.7 & -156.8 & 87.4 \\
\hline 3 & 348.5 & -274.4 & 172.8 & 135.1 & -126.2 & 72.8 & 125.6 & -237.7 & 98.9 \\
\hline 4 & 117.3 & -236.1 & 29.2 & 67.0 & -94.0 & 54.7 & 113.7 & -198.0 & 78.5 \\
\hline 5 & 111.1 & -200.0 & -3.8 & 42.5 & -103.0 & 10.6 & 53.5 & -160.5 & 19.3 \\
\hline
\end{tabular}

Table 4: Calculated internal metacarpophalangeal joint contact forces (Newtons) on the distal end of the metacarpals with the wrist in a neutral position (metacarpal coordinate system - see Figure 3). 


\begin{tabular}{|l|l|l|l|l|l|l|l|l|l|}
\hline & \multicolumn{3}{|c|}{ Neutral [N] } & \multicolumn{3}{c|}{ Radial deviation [N] } & \multicolumn{3}{c|}{ Ulnar deviation [N] } \\
\hline & 1 & 2 & 3 & 1 & 2 & 3 & 1 & 2 & 3 \\
\hline & & & & & & & & & \\
\hline Radiotriquetral & 74.4 & 22.6 & 14.5 & 15.5 & 19.6 & 26.1 & 12.4 & 20.5 & 27.0 \\
\hline Scaphotrapezoid & 231.9 & 51.2 & 158.9 & 87.5 & 313.2 & 55.6 & 105.0 & 360.7 & 0 \\
\hline Scaphotrapezium & 24.9 & 31.6 & 21.6 & 90.3 & 114.9 & 124.0 & 98.8 & 62.1 & 0 \\
\hline Scaphotriquetrum & 0 & 0 & 4.5 & 0 & 0.4 & 1.9 & 0.2 & 2.7 & 0.9 \\
\hline Radiocapitate & 27.1 & 5.9 & 6.5 & 0 & 7.5 & 0.3 & 0.5 & 14.9 & 0.5 \\
\hline Scapholunate & 26.4 & 0.1 & 14.0 & 1.3 & 1.9 & 0 & 4.2 & 0.7 & 19.7 \\
\hline Lunotriquetrum & 0 & 2.8 & 5.7 & 10.4 & 4.2 & 10.3 & 6.3 & 7.0 & 0.6 \\
\hline
\end{tabular}

Table 5: Ligamentous forces predicted in the model for selected ligaments, by subject 13.

\begin{tabular}{|l|l|l|l|l|l|l|l|l|l|}
\hline & \multicolumn{3}{|c|}{ Neutral position [\% ] } & \multicolumn{3}{c|}{ Radial deviation [\%] } & \multicolumn{4}{c|}{ Ulnar deviation [\% ] } \\
\hline & 1 & 2 & 3 & 1 & 2 & 3 & 1 & 2 & 3 \\
\hline & & & & & & & & & \\
\hline Radius & 91.1 & 92.8 & 78.7 & 73.9 & 96.5 & 81.4 & 75.9 & 77.0 & 87.7 \\
\hline Ulna & 8.9 & 7.2 & 21.3 & 26.1 & 3.5 & 18.6 & 24.1 & 23.0 & 12.3 \\
\hline
\end{tabular}

Table 6: Load distribution through the radius and ulna, by subject 1-3. 